\title{
ACOMPAÑAMIENTO DE ESTUDIANTES CON DISCAPACIDAD AUDITIVA EN LA ESCUELA DE CIENCIAS PSICOLÓGICAS USAC
}

\section{ACCOMPANIMENT OF STUDENTS WITH HEARING DISABILITIES IN THE SCHOOL OF PSYCHOLOGICAL SCIENCES USAC}

\section{Referencia del artículo}

Ureta, F., Alvarez, G., Parada, D. y Mencos, M. (2020). Acompañamiento de estudiantes con discapacidad auditiva en la escuela de ciencias psicológicas USAC. Revista Científica del SEP. 3(1), 9-15. DOI: https://doi.org/10.36958/sep.v3i01.26

\begin{abstract}
Francisco José Ureta Morales
fureta@psicousac.edu.gt

Licenciado en Psicología, Mo. en Educación especializado en Currículo, Mo. en Medición, Evaluación e Investigación Educativas, PhDSt en Filosofía

Escuela de Ciencias Psicológicas, Facultad de Humanidades, USAC
\end{abstract}

\section{Gabriela Alvarez García}

galvarez@psicousac.edu.gt

Licenciada en Psicología, Maestra en Análisis Social de la Discapa-cidad

Escuela de Ciencias Psicológicas, Universidad de San Carlos de Guatemala

\section{Doris Melissa Parada}

paradaju10@hotmail.com

Licenciada en Psicología

Escuela de Ciencias Psicológicas, Universidad de San Carlos de Guatemala

María Alejandra Mencos mencosalejandra97@gmail.com

Estudiante de la Licenciatura en Psicología

Escuela de Ciencias Psicológicas, Universidad de San Carlos de Guatemala

Fecha de recibido: $11 / 05 / 2020$

Fecha de aceptado: 02/11/2020

\section{RESUMEN}

La Escuela de Ciencias Psicológicas de Universidad de San Carlos de Guatemala (USAC) creó el Proyecto de Ejercicio Técnico Supervisado (ETS) y Ejercicio Profesional Supervisado (EPS) para que estudiantes de la carrera puedan ser Facilitadores Avanzados de lengua de señas y, acompañen a estudiantes con discapacidad (ECD) como una opción de graduación.

El objetivo general del proyecto es promover la educación inclusiva que permita a los ECD un adecuado proceso de aprendizaje y el desarrollo de las competencias como futuros psicólogos; el proyecto se basa en el modelo social de la discapacidad y entre sus principales logros está ampliar la cobertura de atención, ya que la intérprete contratada no lograba cubrir todas las clases, generar accesibilidad en los procesos de enseñanza y aprendizaje, realización de talleres de concientización con personal administrativo, grabación de videos en lengua de señas e investigación sobre discapacidad en la educación superior.

Como desafíos aumentar el lenguaje técnico en señas y disposición de docentes para realizar los ajustes razonables.

\section{PALABRAS CLAVE}

Educación superior, educación inclusiva, lengua de señas, accesibilidad, ajustes razonables

\section{ABSTRACT}

The School of Psychological Sciences of the University of San Carlos de Guatemala (USAC) created the Project of Supervised Technical Exercise (ETS) and Supervised Professional Exercise (EPS) so that students of the career can be Advanced Facilitators of sign language and, accompany Students with Disabilities (ECD) as a graduation option.

The general objective of the project is to promote inclusive education that allows ECDs an adequate learning process and the development of competencies as future psychologists; The project is based on the social model of disability and among its main achievements is to expand the coverage of care, since the contracted interpreter could not cover all the classes, generate accessibility in the teaching and learning processes, conduct awareness workshops with administrative staff, video recording in sign language, and research on disability in higher education.

As challenges, increase technical sign language and the willingness of teachers to make reasonable adjustments.

\section{KEYWORDS}

Higher education, inclusive education, sign language, accessibility, reasonable accommodation 


\section{I. - INTRODUCCIÓN}

La USAC ha realizado esfuerzos significativos en torno a visibilizar las necesidades de las personas con discapacidad, sin embargo, estos han sido aislados y poco pertinentes. El 14 de octubre de 2014, el Consejo Superior Universitario aprueba la propuesta que reúne el conjunto integral de quince políticas enmarcadas dentro de los ejes funcionales y operativos de la USAC: docencia, investigación, extensión, administración, territorio e infraestructura, planificación y seguimiento.

Su objetivo principal es: asegurar y promover el pleno ejercicio de todos los derechos humanos y las libertades fundamentales de las personas con discapacidad, sin discriminación alguna, creando oportunidades de integración, desarrollo y participación comunitaria en toda la Universidad de San Carlos de Guatemala. Se priorizan las estrategias coherentes, programas y proyectos integrados e integrales de fortalecimiento del desarrollo organizadas en los seis ejes mencionados.

\section{OBJETIVO GENERAL:}

Relacionar las acciones implementadas por la Escuela de Ciencias Psicológicas, con los compromisos asumidos al aprobar las Políticas de Atención a la Población con Discapacidad en la Universidad de San Carlos de Guatemala.

\section{OBJETIVOS ESPECÍFICOS:}

- Identificar las buenas prácticas que se han desarrollado en la Escuela de Ciencias Psicológicas, respecto a la inclusión de estudiantes con discapacidad auditiva.

- Analizar los logros y desafíos que se presentan en el proceso de inclusión de estudiantes con discapacidad auditiva, tanto en el proceso de admisión, ingreso, permanencia y egreso de la universidad.

\section{MARCO TEÓRICO Y CONTEXTUAL}

2. Acceso a la educación superior

En el Artículo 24 de la Convención sobre Derechos de las Personas con Discapacidad (en adelante La Convención) -que el Estado de Guatemala ratificó en 2008-, en el inciso 5, expresa que "Los Estados Partes asegurarán que las personas con discapacidad tengan acceso general a la educación superior, la formación profesional, la educación para adultos y el aprendizaje durante toda la vida sin discriminación y en igualdad de condiciones con las demás. A tal fin, los Estados Partes asegurarán que se realicen ajustes razonables para las personas con discapacidad"; asimismo, en el Artículo 9 declara que "los Estados Partes adoptarán medidas pertinentes para asegurar el acceso de las personas con discapacidad, en igualdad de condiciones con las demás, al entorno físico, el transporte, la información y las comunicaciones, incluidos los sistemas y las tecnologías de la información y las comunicaciones (...)", ONU (2016). Por lo que el Estado de Guatemala debe responder ante esta demanda y la Universidad de San Carlos de Guatemala (en adelante USAC) en su calidad de única universidad estatal del país, tiene corresponsabilidad con el Estado de dar cumplimiento a este instrumento internacional de Derechos.

En cumplimiento a los requerimientos para promover el acceso a una educación superior de calidad para las personas con discapacidad, el 14 de octubre de 2014, el Consejo Superior Universitario aprueba la propuesta que reúne el conjunto integral de quince políticas enmarcadas dentro de los ejes funcionales y operativos de la USAC: docencia, investigación, extensión, administración, territorio e infraestructura, planificación y seguimiento (Alvarez y Ureta, 2017; Carrera y Ureta, 2017).

La USAC ha emprendido algunas acciones tales como: la construcción o acondicionamiento de rampas en espacios de articulación y de acceso a edificios, remodelación de ciertas áreas tanto en el campus central como en algunos centros universitarios (USAC, 2014); se ha conformado la Comisión Universitaria para la Atención a la Población con Discapacidad en la USAC, que ha sido un logro de vital importancia (USAC, 2016).

También se han realizado otras acciones como: elaboración de Trabajos de graduación y proyectos e investigaciones que abordan diversas aristas que conforman la discapacidad, realización de procesos de formación en discapacidad organizados a lo interno de las unidades académicas y desde la Dirección de Bienestar Estudiantil, la contratación de una profesional encargada de acompañar a los estudiantes de primer ingreso para la realización del proceso de inscripción y recientemente se ha conformado una sub comisión para la adaptación de las pruebas de primer ingreso, para que estén en formato accesible, proceso que está dando los primeros pasos.

Sin embargo, aún son pocas las acciones para responder de manera efectiva a la población con discapacidad en la USAC, lo cual demanda una propuesta integral, que sea pensada desde la administración central, las unidades académicas y otras instancias que abarque a toda la población, USAC (2014).

Las autoridades de la Escuela de Ciencias Psicológicas (en adelante ECPs) de la USAC, realizan esfuerzos sistemáticos y sostenidos para promover una educación inclusiva, dando cumplimiento así a las Políticas de Atención a la Población con Discapacidad. 
Claros de que la discapacidad es compleja y está conformada por diversas aristas desde las que se debe analizar, ha focalizado en un primer momento los ajustes razonables para hacer accesible el proceso de enseñanza y aprendizaje, específicamente para los estudiantes con discapacidad auditiva. La intención es que se desarrollen en igualdad de condiciones y la relevancia que tiene el abordaje del tema radica en que gran parte del éxito que tengan los estudiantes con discapacidad en su tránsito por la universidad y su egreso, está relacionado con la accesibilidad que tengan tanto en el aspecto físico, como de información y comunicación durante su permanencia en los salones de clase. Como parte de estas acciones, determinaron la contratación de la Licenciada en Psicología Doris Melissa Parada, ella es una intérprete profesional de lengua de señas, actualmente es la presidenta de la Asociación Nacional de Intérpretes para Sordos y Sordociegos -ANDISYSC-.

Sin embargo, la Licenciada Parada no lograba cubrir la demanda para la interpretación efectiva de las clases, por lo que las autoridades designaron (por medio del oficio-CGD-008-2018 del 12 de febrero del 2018 de la Dirección de la Escuela de Ciencias Psicológicas) la elaboración de la convocatoria y creación del Proyecto de ETS para estudiantes de Carreras Técnicas. Estos estudiantes deberían ser Facilitadores de Lengua de Señas (LENSEGUA), interesados en realizar acompañamiento a estudiantes con discapacidad (ECD) de esta Unidad Académica. La responsabilidad cayó en el Maestro Francisco José Ureta del Departamento de EPS, quien desde marzo de 2018 ha trabajado en conjunto con la Maestra Gabriela Alvarez, Coordinadora de la carrera del Profesorado de educación especial (PEE) y la Licenciada Parada, en la conformación y coordinación de dicho proyecto.

\subsection{PROYECTO DE ETS Y EPS EN LA ECPS}

Este proyecto emerge de la urgencia de ofrecer acompañamiento e interpretación de clases a ECD auditiva y apoyo a ECD visual de la Escuela de Ciencias Psicológicas, quienes en anteriores momentos lo han solicitado a las autoridades académicas. El presente proyecto es la respuesta a dicha solicitud, enfrentando limitantes serias como lo es falta de presupuesto para la contratación de otra intérprete y que tenga la experticia en la carrera, así como no lograr cubrir la demanda actual. El objetivo general de este novedoso proyecto es promover la educación inclusiva en la Escuela de Ciencias Psicológicas, por medio del acceso a la información para los estudiantes con sordera, que permita un adecuado proceso de aprendizaje y el desarrollo de sus competencias como futuros psicólogos, con el acompañamiento de estudiantes que realizan el ETS del PEE o EPS de la Licenciatura en Psicología.

La orientación del proyecto se basa en el modelo social de abordaje de la discapacidad que enfatiza la autonomía, la integración, las capacidades y la realización de los ajustes razonables, así como la interacción del ECD con la infraestructura y el ambiente donde vive, considerando las determinantes sociales que influyen y condicionan la discapacidad (Vargas y Ureta, 2017).

El proyecto se enmarca en el enfoque del marco lógico e incluye el análisis de involucrados, problemas, objetivos y posibles soluciones al problema priorizado. Se han formulado los objetivos, indicadores, actividades y medios de verificación del proyecto, para que sea factible verificar los avances y dificultades en su ejecución.

Se planteó un cronograma anual, el cual es la base para su trabajo en cada año de ejecución del proyecto. Incluye un presupuesto inicial y de mantenimiento para el proyecto, la inversión inicial en materiales y tecnología sería elevada, pero quedarían para ser utilizados por futuras promociones de ECD. (Ureta y Alvarez, 2019).

El primer paso para la formulación del proyecto fue tener una idea precisa de los ECD por atender, el siguiente cuadro incluye la información de los 17 estudiantes que solicitaron su ingreso a la ECPs, 7 para el 2018 y 10 para el 2019.

Predomina la discapacidad visual, la auditiva y la física, así como la inscripción en la licenciatura en psicología y PEE, genera diversos desafíos para su adecuada atención.

Sin embargo, al no tener una oficina específica de atención a los ECD se dificulta su apropiada identificación y seguimiento ya en la ECPs, ya que esta es la información que solicitaron exonerarse de los exámenes de admisión.

Cuarirs 1

Estudiuntes exonerados de pruehas de ingreso ciclos 2018 y 2019, ECP:

\begin{tabular}{|c|c|c|c|}
\hline Año & 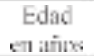 & Disermeidad & Carrers \\
\hline 2018 & 20 & Fisica & $\begin{array}{c}\text { Profewotado en Education Espocial y } \\
\text { Liccnciatura en Psicologia }\end{array}$ \\
\hline 2018 & 25 & Visual & $\begin{array}{l}\text { Tesnico en orientacion vocicional y } \\
\text { laboral. Licenciatura en pricologia }\end{array}$ \\
\hline 2018 & 20 & Y'jsual & Licensiatura in psicologia \\
\hline 2018 & 20 & Auditiva & Profesorado en educicion especial \\
\hline 2018 & 20 & Fisica & Licenciatura en psicolingia \\
\hline $201 \mathrm{~K}$ & 20 & Fisica & Licenciatura en psicolvogia \\
\hline 2018 & 20 & Fisica & Licenciatura en psicologia \\
\hline 2019 & 18 & Visual & Licenciatura en psionlogin \\
\hline 2019 & 21 & Visual & Lisenciatura en psioglegia \\
\hline 2019 & 28 & Visual & Licenciatura en psicologia \\
\hline 2019 & 22 & Visual & Licenciatura en psicologia \\
\hline 2014 & 25 & Visual & Licenciatura en psicolingia \\
\hline 2019 & I8 & Visual & Profesorader en educación especial \\
\hline 2019 & 21 & Mültiple (fisica, visalal) & Profesorado en educacion especial \\
\hline 2019 & 30 & Muthiple (fisica, visual) & Profesorado en educación especial \\
\hline 2019 & 21 & Fisica & Profesorado en cducación espocial \\
\hline 2019 & 40 & Auditiva & Liscnciatura en psicologia \\
\hline
\end{tabular}


El ETS y el EPS lo realizan estudiantes con cierre de pensum de Carrera Técnica o Licenciatura, en instituciones públicas, privadas y sin fines de lucro. Su filosofía parte de la congruencia con la Dirección General de Extensión de la USAC, la cual busca fortalecer la integración Universidad-sociedad, la ciencia y la cultura en todas sus manifestaciones, así como la identidad nacional, por medio de la promoción de la salud mental y psicológica (las personas son conscientes de sus capacidades, por lo que pueden afrontar las tensiones normales de la vida cotidiana, pueden trabajar de forma productiva y son capaces de preocuparse y hacer contribuciones a su comunidad).

También se pretende desarrollar capacidades institucionales para contribuir al estudio de propuestas de solución a problemas psicosociales, para el desarrollo de la nación guatemalteca en los diferentes lugares e instituciones donde se realizan estos procesos de graduación de carrera técnica o de licenciatura en psicología. Se realiza por medio del diseño e implementación de proyectos de intervención psicológica que contribuyan al desarrollo y mejoramiento de la salud mental y psicológica de la población guatemalteca, mediante el diagnóstico, la propuesta y ejecución del Proyectos con participación comunitaria y ajustada a las necesidades institucionales.

Este proceso de graduación implica el desarrollo de tres ejes de trabajo:

1. Atención individual: al inicio se identifican los principales problemas psicosociales de la población a atender, con este elemento se atienden individualmente dichas problemáticas, principalmente con procesos psicoterapéuticos.

2 Prevención: realización de talleres psicoeducativos que buscan informar a las personas y orientarlas sobre temáticas asociadas a sus problemas psicosociales e institucionales, para que se busquen las estrategias para la prevención de dichos problemas identificados.

3. Investigación: se realiza la investigación de problemas identificados y que necesiten de este proceso para su resolución, la idea es que los resultados obtenidos permitan coadyuvar a la solución de dicha problemática con el conocimiento nuevo generado.

En este proyecto se han realizado las siguientes acciones de acompañamiento de estudiantes con sordera principalmente:

1. Atención individual: traducción de las clases a ECD, apoyarlas en la comunicación con sus pares estudiantiles y docentes, para que la información la reciban apropiadamente y puedan generar los aprendizajes esperados. También el trabajo individual con cada estudiante para aumentar su vocabulario, de manera que lo discutido en clase sea apropiadamente analizado por los ECD.

2 Prevención: realización de un taller de concientización con el personal administrativo de la ECPs para la apropiada atención de los ECD, así como la generación de unos videos con lengua de señas con contenido asociado a la ECPs y explicación a los docentes de la situación de los ECD para que realicen las adecuaciones curriculares respectivas.

3. Investigación: glosarios técnicos específicos de la carrera con lengua de señas desde la propia interpretación de los ECD y consulta de menús a las cafeterías del CUM para adecuarlos a los ECD.

Dentro de las experiencias en proceso del ETS se citan que hay diferentes actitudes por parte de docentes y de compañeros, tanto a favor de los procesos de inclusión como en disenso, principalmente por que se cree que el estudiante con discapacidad no alcanzará el nivel de competencias necesario para egresar de una carrera universitaria. Por otro lado, se anotan algunos elementos con base a la experiencia, sobre las destrezas que las ECD auditiva han desarrollado al contar con intérprete.

En general se observa que el acompañamiento a las estudiantes ha sido enriquecedor, lleno de muchas experiencias y retos que cada día se van descubriendo y resolviendo con base a necesidades de las estudiantes, buscando siempre la equidad al contar con intérprete de lengua de señas.

El acceso a la información es importante para comprender la totalidad del contenido que se imparte, la identificación que tienen las estudiantes con la intérprete es fundamental ya que con esto se logra un avance específico en cuanto al desenvolvimiento de cada una. Su participación ha sido activa, se sienten incluidas, tienen una mejor capacidad de análisis y síntesis de los contenidos, las destrezas de aprendizaje mejoraron significativamente, cabe mencionar que es básico tener una interacción constante.

La realización del ETS favoreció acercarse a las estudiantes y conocerlas de mejor forma, lo cual ha permitido desarrollar técnicas y herramientas de comunicación eficaz, se mejoró la comprensión de cada tema, con ellas se ha desarrollado un vocabulario de señas relacionadas a la Psicología y sus componentes que antes no tenían y hacían difícil la comprensión de conceptos de la disciplina psicológica. 
En cuanto a los compañeros de estudio sin discapacidad, están sensibilizados y conocen el tema, se observa la disposición de interactuar y aprender la lengua de señas como medio de comunicación con las estudiantes con discapacidad auditiva, según las observaciones de clase que realizó la estudiante cuando realizó su ETS en apoyo a las ECD auditiva (Mencos, 2019). Algunos han manifestado su interés de aplicar al programa de ETS, la cual es de mucha ayuda para el intérprete oficial y el proyecto analizado.

En relación a los docentes, la mayoría evidencian disposición para la accesibilidad en realizar las adecuaciones curriculares y ajustes razonables para las estudiantes con sordera. Otros que ignoran totalmente el tema, han mostrado actitudes inadecuadas, lo que hace que no estén de acuerdo de realizar los cambios necesarios para una inclusión de las estudiantes. Pero esta situación se enfrenta al mismo tiempo se va caminando en el proyecto, resolviendo y orientando a través de la de diferentes actividades de sensibilización y concientización como parte del programa de ETS (Estrada y Ureta, 2017).

Dentro de la experiencia de la intérprete de lengua de señas en la ECPs, enfatiza que no es de traducir como lo hace Google, sino que lleva consigo una serie de elementos, como: el conocimiento previo a los temas que se imparten, la cultura de la persona sorda, los gestos, las configuraciones de las manos, las polisemias y ver cual se utiliza dependiendo el contexto y así poder introducirlo en el momento adecuado, para una comprensión asertiva de los temas abstractos y subjetivos que tiene la Psicología, esto se da voz a señas. Cuando se interpreta de señas a voz conlleva los elementos ya mencionados, a ello se agrega la gramática, estructuración y glosa de la persona sorda; la cual requiere de un esfuerzo doble para poder interpretar lo más claro y cercano a lo que la persona con discapacidad auditiva quiere expresar.

\section{ANÁLISIS DE RESULTADOS}

En el siguiente cuadro se resumen los principales aportes del proyecto analizado, los cuales consisten en las buenas prácticas identificadas, desafíos y posibles propuestas de solución.

\begin{tabular}{|c|c|c|}
\hline Buenas prócticas & Desatios & Propuestas \\
\hline $\begin{array}{l}\text { 1.La ECT's forma parte de la } \\
\text { subcomision de adaptación } \\
\text { de las pruchas de ingrew. }\end{array}$ & 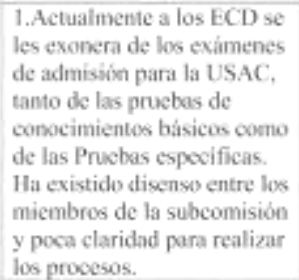 & $\begin{array}{l}\text { 1.Clarificar los procesos que } \\
\text { se desamollan para realizar } \\
\text { la adantacion de las Pructas } \\
\text { de ingreso a formatos } \\
\text { accesibles para ECD. }\end{array}$ \\
\hline $\begin{array}{l}\text { 2. Identificación y } \\
\text { acompañamiento de } \\
\text { estudiancs con discapacidad } \\
\text { a la ECPs. }\end{array}$ & $\begin{array}{l}\text { 2.A bo interno do la ECPS no } \\
\text { hay un departamento } \\
\text { unidad encargada de darle } \\
\text { scguimicnto a los ECD que } \\
\text { hán ingresado. Luego de la } \\
\text { presentaciòn de la poocncia }\end{array}$ & $\begin{array}{l}\text { 2.Generar las acciones desde } \\
\text { las autoridades para abrir la } \\
\text { unidad de apoyo al ECD, } \\
\text { que sea responsable de } \\
\text { acompañar los procesos } \\
\text { inclusivos y redirigir las }\end{array}$ \\
\hline
\end{tabular}

a

totalk individualizado.

accesible y acorde a bo

productos que venden.

\section{Se ha fortalecido la} invextigación sobre discapacidad en les trabajos de graduación tanto de pre grado como de grado. Algunos revisores se han involucrado en procesos de formacion.

Bucnas practicas 10. Sc abrió la Masstria en Análisis Social de la Dixcapacidad (MASDIS). única co Guatemala y segunda en la región centroamericanta, coresaroo los $17 \mathrm{de}$ la $1^{\mathrm{a}}$ cohorte en 2017.

utifizarlo ch sus establecimientos.

9. Algunos de los revisores de los trabsjos de graduación no sc han el desarrollo del proceso.

\section{Desatios} 10. En cste anto sc abrie la sejaunda cohorte de la MASDIS y se podria formado en el tema, lo cual erea una seria limitante para vincular con este proceso de ETS y EPS. administrativo, ya que manifestaron que no sabian como comunicarsc con las ECD auditivn.

9. Generar proceson sistemáticos de formación co discapacidad y mantencer la ancrtura de la Camera de PEE para intercambiar aportes sobre el tema. Propuestas 10. Establecer relaciones de eqle proceso de ETS y EPS con la MASDIS. 


\section{CONCLUSIONES}

Con base en las evidencias y análisis realizados, se formulan las siguientes conclusiones:

1. Las acciones implementadas por la Escuela de Ciencias Psicológicas, responden a los compromisos asumidos por la USAC al aprobar las Políticas de Atención a la Población con Discapacidad dentro de la universidad.

2 La contratación de la profesional intérprete fue fundamental para iniciar el proceso de la educación universitaria inclusiva en la ECPs con las 4 ECD auditiva atendidas en 2019.

3. Existen pasos importantes que se pueden definir como buenas prácticas que se han desarrollado en la Escuela de Ciencias Psicológicas, respecto a la inclusión de estudiantes con discapacidad auditiva. Tales como contratación de traductora de lengua de señas, aumento de proceso de formación en discapacidad, interpretación de las clases a ECD auditiva, adecuaciones curriculares, aumento de investigación sobre discapacidad, ofrecer estudios de maestría e involucrar a las 5 carreras técnicas.

4 Se han identificado logros y desafíos que se presentan en el proceso de inclusión de estudiantes con discapacidad auditiva, tanto en el proceso de admisión, ingreso, permanencia y egreso de la universidad, para los cuales se han planteado algunas propuestas de abordaje. Tal como se citó en el cuadro 2: identificación y acompañamiento de ECD auditiva, contratación de la intérprete de Lengua de señas, censo de $E C D$, interpretación en Lengua de señas en los salones de clase, orientación a docentes, fortalecimiento de la investigación sobre la discapacidad y apertura de la MASDIS.

5 Entre los principales desafíos se indican que la interprete no se alcanza para atender a todas l las clases de las ECD auditiva, limitantes de presupuesto, sistematizar más los esfuerzos realizados, aumentar el vocabulario abstracto de las ECD, docentes que se oponen a las adecuaciones curriculares, relacionar la MASDIS con este proceso de ETS y EPS y falta de formación a docentes y revisores de tesis sobre discapacidad.

\section{RECOMENDACIONES}

Con base en las anteriores conclusiones se postulan las siguientes recomendaciones:

6. Continuar con el proyecto de ETS y EPS para estudiantes en proceso de graduación de la ECPs, como opción viable e inclusiva.

7. Sistematizar y profundizar la formación docente sobre el tema de la discapacidad, principalmente en la comunicación y evaluación de los aprendizajes.

\& Gestionar y asignar más fondos del presupuesto de la ECPs para la educación inclusiva.

9. Diseñar e implementar una oficina de atención a las PCD en la ECPs, desde que solicitan su ingreso y exoneración de pruebas de primer ingreso, en su desarrollo ya en la formación profesional y seguimiento y apoyo a sus necesidades.

\section{REFERENCIAS BIBLIOGRÁFICAS}

Alvarez, G., \& Ureta, F. (2017). Construcción Metodológica del Eje de Docencia -incisos 2 y 4 - de las Políticas de Atención a la Población con Discapacidad de la Universidad de San Carlos de Guatemala, en la Escuela de Ciencias Psicológicas y la Escuela de Ciencia Política. Guatemala: Tesis de maestría, Universidad de San Carlos de Guatemala.

Carrera, K., \& Ureta, F. (2017). Inclusión Educativa en la Educación Superior: Actitud Docente y Experiencias Estudiantiles. Guatemala: Tesis de maestría, Universidad de San Carlos de Guatemala.

Elizondo, I. (2019). Información de personas con discapacidad que solicitaron su exoneración de los exámenes de admisión. Guatemala; USAC, Bienestar estudiantil.

Estrada, V., \& Ureta, F. (2017). Procesos de inclusión laboral para docentes que presentan discapacidad motriz en la Universidad de San Carlos de Guatemala. Guatemala: Tesis de maestría, Universidad de San Carlos de Guatemala

Organización de Naciones Unidas. (2006). Convención Internacional sobre Derechos de las Personas con Discapacidad.Convención. Paris: ONU. 
Mencos, M. (2019). Proyecto de Factibilidad. El intérprete deLengua de Señas como facilitador de los procesos educativos superiores en la Escuela de Ciencias Psicológicas USAC.

Universidad de San Carlos de Guatemala. (2014). Comisión Universitaria para la Atención a la Población con Discapacidad. Guatemala: USAC, Coordinadora General de Planificación.

Ureta, F. \& Alvarez, G. (2019). Proyecto de Ejercicio Técnico Supervisado o Ejercicio Profesional Supervisado de Acompañamiento a estudiantes con discapacidad en la Escuela de Ciencias Psicológicas. Guatemala: USAC.

Vargas, V. \& Ureta, F. (2017). La identificación de personas con discapacidad en áreas rurales del departamento de Sololá. Guatemala: Tesis de maestría, Universidad de San Carlos de Guatemala.

\section{Sobre los autores}

\section{Francisco José Ureta Morales}

Es Profesor de licenciatura y maestría en la USAC desde hace diez años, investigador y evaluador de procesos psicoeducativos, cierre de pensum del doctorado en filosofía por la URL, maestrías en educación especializada en currículo $y$, en medición, evaluación e investigación educativas por la UVG, psicólogo por la USAC, ha publicado diversos artículos en revistas indexadas internacionales y nacionales, líneas de investigación principales: evaluación de aprendizajes y de programas educativos, psicometría y epistemología.

\section{Gabriela Alvarez García}

Es Coordinadora de la Maestría en Análisis Social de la Discapacidad y la carrera técnica del Profesorado en Educación Especial, que se desarrollan en la Escuela de Ciencias Psicológicas de la Universidad de San Carlos de Guatemala. Profesora del área de Postgrado, Licenciatura en Psicología y Carreras Técnicas. Psicóloga egresada de la Universidad de San Carlos de Guatemala. Analista Transaccional certificada miembro clínico, por la Asociación Latinoamericana de Análisis Transaccional -ALAT. Consultora independiente en temáticas de educación y discapacidad.

\section{Doris Melissa Parada}

Es psicóloga, Terapista de Lenguaje en la Universidad de San Carlos de Guatemala, cursando el 3er semestre de la Maestría en Análisis Social de la Discapacidad, Intérprete Profesional de Lengua de Señas (desde hace 25 años) lengua de señas como primer idioma adquirido en el hogar (lengua materna). Presidenta de la Asociación Nacional de Intérpretes para Sordos y Sordociegos ANDYSISC. Intérprete Oficial de la Escuela de Ciencias Psicológicas y del Noticiero dominical del canal Guatevisión.

\section{María Alejandra Mencos}

Es Educadora Especial, Cierre de Pensum Licenciatura en Psicología, Maestrante de la Maestría en Análisis Social de la Discapacidad, educadora inclusiva de niños y jóvenes en condición de discapacidad, formada como Intérprete de Lengua de Señas guatemalteca en un contexto de aprendizaje social, Fundadora de Integra Guatemala.

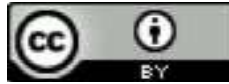

Este texto está protegido por una licencia Creative Commons 4.0.

Usted es libre para Compartir - copiar y redistribuir el material en cualquier medio o formato $-\mathrm{y}$ Adaptar eldocumento - remezclar, transformar y crear a partir del material- para cualquier propósito, incluso comercialmente, siempre que cumpla la condición de:

Atribución: Usted debe reconocer el crédito de una obra de manera adecuada, proporcionar un enlace a la licencia,e indicar si se han realizado cambios. Puede hacerlo en cualquier forma razonable, pero no de forma tal que sugieraque tiene el apoyo del licenciante o lo recibe por el uso que hace. 